\title{
ENSO组合模态对夏季西北太平洋异常反气旋 维持的关键作用
}

${\text { 张文君 }{ }^{1^{*}} \text {, 余佐励 }{ }^{1,2}, \text { 姜枫 }{ }^{1}, \text { 耿新 }^{1} \text {, 张人禾 }}^{3}$

1. 南京信息工程大学气象灾害教育部重点实验室/大气科学学院, 南京 210044;

2. 张家港市气象局, 苏州 215600 ;

3. 复旦大学大气与海洋科学系/大气科学研究院, 上海 200438

* 通讯作者, E-mail: zhangwj@nuist.edu.cn

收稿日期: 2021-10-12; 收修改稿日期: 2022-02-04; 接受日期: 2022-02-18; 网络版发表日期: 2022-06-13

国家自然科学基金项目(批准号: 42125501、42088101)资助

摘要 基于1979 2020年美国环境预报中心/美国大气研究中心(NCEP/NCAR)提供的逐月大气环流和美国国家大 气海洋管理局(NOAA)提供的逐月海表温度资料, 文章研究了厄尔尼诺(El Niño)衰减年夏季西北太平洋异常反气 旋(WNPAC) 维持的关键物理成因, 对比分析了西北太平洋(WNP)冷海温、印度洋(IO)暖海温和ENSO组合模态在 其中的作用. 结果表明, El Niño衰减年冬、春季, WNP地区通常存在显著的冷海温异常, 但该信号不能持续到夏 季, 因而不能很好地解释El Niño衰减年夏季WNPAC的维持. IO暖海温对El Niño衰减年夏季WNPAC的影响具有 明显的年代际差异, 2000 年之前, IO暖海温能通过斜压大气开尔文波响应显著加强WNPAC, 但 2000 年之后, 这种 影响较弱。进一步分析发现，这种影响的年代际减弱可能与ENSO事件衰减速度的加快有关，2000年之后的E1 $\mathrm{Niño}$ 事件衰减较快, 衰减年夏季热带中东太平洋表现为类La Niña型海温异常, 热带IO上没有显著的暖海温异常, 因而其对El Niño衰减年夏季WNPAC的影响较弱. 与WNP冷海温和IO暖海温相比, ENSO组合模态与夏季WNPAC 的关系在研究时段一直较为稳定, 在El Niño衰减年夏季WNPAC的维持中起到了关键的作用. 由于ENSO组合模 态自身持续性较强, 因此基于 ENSO组合模态可以更好地预测北半球夏季的WNPAC及相关天气气候异常.

关键词 ENSO, 西北太平洋异常反气旋, ENSO组合模态

\section{1 引言}

作为热带海气耦合年际变率的最主要模态，厄尔 尼诺和南方涛动(El Niño and Southern Oscillation, ENSO)对全球气候都能产生重要影响(Bjerknes，1969;
Wallace和Gutzler，1981; Wallace等，1998; Alexander 等，2002), 其中对东亚地区的气候影响也非常显著 (Zhang等，1996；陶诗言和张庆云，1998; Chen等, 2000; Wang等, 2000). 研究表明, 西北太平洋(WNP)异 常反气旋(WNPAC)/气旋(WNPC) 是ENSO影响东亚气 
候的重要纽带, 它一般从ENSO发展年秋季开始建立, 在衰减年春季达到鼎盛状态，并一直持续至初夏(Harrison和Larkin, 1996; Wang等, 2000; 张人禾等, 2017). 在El Niño成熟期, 受WNPAC西北侧异常西南暖湿气 流的影响, 东亚冬季风容易偏弱(Zhang等, 1996; Wang 等，2003)，中国东南部降水容易偏多(Zhang等，1999; Zhang和Sumi, 2002; Wang等, 2017). 在El Niño衰减年 夏季, WNPAC的位置有所北抬, 中国长江中下游地区 梅雨降水增强(Chang等, 2000a, 2000b; Lin和Lu, 2009; 黄平和黄荣辉, 2010; $\mathrm{Li}$ 等, 2017; 张人禾等, 2017). 当 La Niña事件发生时，情况虽然并不是反对称，但大致 相反(Zhang $\mathrm{R} \mathrm{H}$ 等, 2015). 为了描述方便, 本文主要以 El Niño期间的WNPAC为例，对于La Niña期间的 WNPC, 只需从相反位相考虑即可.

鉴于WNPAC的重要性, 其生成和维持机制一直是 学术界的研究热点. Zhang等(1996，1999)指出当赤道 中东太平洋ENSO事件发生时, 西北太平洋(WNP)出现 冷海温异常, 局地对流活动受到抑制, 由此产生的大气 Rossby波响应使得WNP副热带高压西伸，导致东亚地 区降水异常. Wang等(2000)提出WNPAC是西太平洋大 气对局地冷海温的响应, 它之所以能长期维持与风-蒸 发-海温正反馈有关, 其机制是: 北半球冬季WNP盛行 东北信风, 当El Niño事件发生时, 赤道东太平洋暖海 温会在其西北侧激发气旋性环流异常(Matsuno, 1966; Gill，1980)，其西部的东北风异常与气候态东北信风 叠加，使风速增强、向上的潜热通量增加，从而导致 西太平洋地区的海温变冷进而在其西侧激发出反气旋 性环流, 其东侧的偏北气流进一步使得信风增强, 海温 进一步变冷, 由此使得WNPAC一直维持到El Niño衰 减年的初夏.

然而, 到El Niño衰减年夏季, WNP局地冷海温的 信号已经消失, 这似乎意味着风-蒸发-海温正反馈机制 对夏季WNPAC作用较弱. 随后, 大量的研究开始从印 度洋(IO)海温的角度(称为印度洋电容器效应) 解释夏 季WNPAC的维持(周天军等, 2004; 黄刚和胡开明, 2008; Xie等, 2009; Du等, 2009; Wu等, 2010), 也有研 究强调了印度洋-西北太平洋联合效应(Indo-western Pacific Ocean Capacitor, IPOC)对WNPAC维持的关键 作用(Wang等, 2013; Xie等, 2016; Wang等, 2018, 2020). El Niño衰减年IO暖海温异常在春季达到峰值并一直 持续到夏季(Klein等，1999). Yang等(2007)和Xie等
(2009)进一步指出, El Niño衰减年IO变暖对WNPAC起 到重要作用, 这种热带 $\mathrm{IO}$ 暖异常海温将会激发出向东 传播的Kelvin波, 而由于边界层的摩擦作用使得在热 带西太平洋两侧产生Ekman辐散, 抑制了WNP对流潜 热的释放, 最终有利于WNPAC的维持. 但是, 最近的 研究发现, IO延迟增暖无法解释对称Kelvin波作用下 WNP大气响应不对称性现象(Stuecker等, 2015). 因此, ENSO期间WNPAC形成和维持的物理机制仍存在一些 函待解决的争议点. 近年来, Stuecker等(2013)在赤道太 平洋低层环流场中发现并定义了 $\mathrm{ENSO}$ 组合模态 (Combination Mode, C-mode), 该模态具有 10 和 15 个月 左右的主周期, 在物理上来源于ENSO年际变率与海温 年循环之间的非线性相互作用，在空间型上则表现为 中太平洋赤道南侧的西风异常和西北太平洋上的反气 旋性环流(McGregor等, 2012, 2013; Stuecker等, 2013; 李海燕等, 2016; Zhang W J等, 2015, 2016a, 2016b). 近 年来, C-mode也被认为是WNPAC形成和维持的重要 机制之一(Stuecker等, 2015; Zhang等, 2016a, 2016b).

综上所述, 前人主要提出了三种WNPAC形成和维 持的可能物理机制: 局地海气相互作用、印度洋电容 器效应和 C-mode, 那么哪个机制对夏季WNPAC的维 持起到关键作用? 本文将基于美国环境预报中心/美国 大气研究中心(NCEP/NCAR)和美国国家海洋和大气 局(NOAA) 提供的最新逐月大气环流和海表温度资料, 采用相关回归、合成分析的方法，对这一问题进行系 统研究, 即对WNP冷海温、IO暖海温和C-mode与北 半球夏季WNPAC的关系分别进行再研究.

\section{2 资料和方法}

本文使用的资料包括: (1) NOAA月平均海表温度 扩展重建资料(ERSST.v5)(Huang等，2017), 水平分辨 率为 $2.0^{\circ} \times 2.0^{\circ}$. (2) $\mathrm{NCEP} / \mathrm{NCAR}$ 全球逐月位势高度 场、风场再分析资料(NCEP/DOE Reanalysis II )(Kanamitsu等, 2002), 水平分辨率为 $2.5^{\circ} \times 2.5^{\circ}$. (3) 美国国 家气候预测中心(Climate Prediction Center, CPC)逐月 降水资料(CMAP)(Xie和Arkin, 1997), 水平分辨率为 $2.5^{\circ} \times 2.5^{\circ}$. 以上资料均取 1979 年 1 月至 2020 年 12 月. 文 中的春季和夏季分别定义为 $3 \sim 5$ 月和 $6 \sim 8$ 月的平均, 冬 季定义为当年12月至次年2月的平均, 如1960年冬季 为1960年12月至1961年2月的平均. 
参照前人的做法, 本文将北半球夏季WNPAC指数 定义为WNP地区 $\left(15^{\circ} \mathrm{N} \sim 25^{\circ} \mathrm{N}, 115^{\circ} \mathrm{E} \sim 150^{\circ} \mathrm{E}\right) 850 \mathrm{hPa}$ 位 势高度场距平的区域平均(Wang等, 2013), 利用区域平 均的流函数定义WNPAC指数可以得到相似结论. $\mathrm{WNP}$ 海温指数定义为WNP地区 $\left(0^{\circ} \mathrm{S} \sim 15^{\circ} \mathrm{N}, 120^{\circ} \mathrm{E} \sim\right.$ $160^{\circ} \mathrm{E}$ )海温距平的区域平均(Wang等, 2013). IO海温 指数定义为印度洋 $\left(10^{\circ} \mathrm{S} \sim 10^{\circ} \mathrm{N}, 50^{\circ} \mathrm{E} \sim 110^{\circ} \mathrm{E}\right)$ 海温距平 的区域平均(Wang等, 2013), 使用北印度洋 $\left(0^{\circ} \sim 10^{\circ} \mathrm{N}\right.$, $\left.50^{\circ} \mathrm{E} 110^{\circ} \mathrm{E}\right)$ 海温距平的区域平均, 文章的定性结论不 会改变. C-mode指数用赤道太平洋 $\left(10^{\circ} \mathrm{S} \sim 10^{\circ} \mathrm{N}, 100^{\circ} \mathrm{E}\right.$ $\sim 80^{\circ} \mathrm{W}$ ) 表面风场经验正交函数分解(EOF)第 2 模态所 对应的标准化时间序列(即PC2)表示.

本文使用的方法包括EOF分解、相关分析和回归 分析等. 采用 CPC 对 ENSO事件的定义标准来節选 ENSO事件, 得到的ENSO事件如表1所示. 需要指出的 是, 由于本文主要关注ENSO衰减年夏天，对于连续 $\mathrm{ENSO}$ 事件，只挑选其最后一年. 鉴于本文主要关注年 际尺度上WNPAC的维持机制, 所有数据均进行了去趋 势处理, 并使用Butterworth带通滤波器对数据进行了 $6 \sim 120$ 个月的滤波.

\section{ENSO衰减年夏季WNP环流特征及其东 亚降水响应}

我们首先给出了1979 2020年夏季WNPAC指数 和前冬Niño-3.4指数的时间序列(图1), 由此可见, 夏季 WNPAC指数具有显著的年际变化特征，大致存在2 5 年的变化周期(图略). 在El Niño事件的衰减年夏季, WNP上一般存在反气旋性环流，对应WNPAC指数为 正，La Niña事件的衰减年夏季则相反(Wang等, 2000; Xie等, 2009; Stuecker等, 2015). 统计上前冬Niño-3.4指 数与夏季WNPAC指数存在 0.53 的相关, 超过了信度为 0.05 的显著性检验，ENSO是夏季WNPAC年际变率的 主要可预报性来源.

夏季WNPAC变率对中国天气气候有着重要的影 响. 我们将夏季WNPAC指数回归到同期 $850 \mathrm{hPa}$ 异常降 水场和风场(图2), 从中可以看出, 当WNPAC指数为正 时，反气旋存在于WNP菲律宾东北部上空，主体范围 大致位于 $15^{\circ} \sim 25^{\circ} \mathrm{N}, 115^{\circ} \sim 150^{\circ} \mathrm{E}$, 其西侧甚至可以延伸 到印度半岛以东的孟加拉湾，在孟加拉湾形成单独的 异常反气旋中心，形成横跨WNP到孟加拉湾更大范围
表 1 1979 2020年ENSO事件

\begin{tabular}{|c|c|}
\hline 事件 & 年份 \\
\hline 厄尔尼诺 & $\begin{array}{ccc}1979 / 1980 、 1982 / 1983 、 1986 / 1987 、 1987 / 1988 、 \\
1991 / 1992 、 1994 / 1995 、 1997 / 1998 、 2002 / 2003 、 \\
2004 / 2005 、 2006 / 2007 、 2009 / 2010 、 2014 / 2015 、 \\
2015 / 2016 、 2018 / 2019\end{array}$ \\
\hline 拉尼娜 & $\begin{array}{c}1983 / 1984 、 1984 / 1985 、 1988 / 1989 、 1995 / 1996 、 \\
1998 / 1999 、 1999 / 2000 、 2000 / 2001 、 2005 / 2006 、 \\
\text { 2007/2008、2008/2009、2010/2011 } \\
\text { 2011/2012、2016/2017、2017/2018 }\end{array}$ \\
\hline
\end{tabular}

的异常反气旋环流(Xie等, 2016). 该环流系统对东亚 地区的气候异常具有显著影响. 当WNPAC指数为正 时，东亚地区存在“南负北正”的降水异常结构, 即中 国长江流域、华北地区以及日本和朝鲜半岛等地降水 明显偏多,而WNPAC中心控制的菲律宾、中南半岛以 及中国华南沿海等地降水显著偏少. 就中国而言, 显著 的降水异常位于长江中下游流域, 这对应于当地梅雨 降水的增多增强，进一步凸显了WNPAC对中国夏季 气候影响的关键作用.

\section{WNPAC 的形成机制探讨}

为了探究夏季WNPAC的形成和维持机制, 我们给 出了夏季WNPAC指数回归的前期冬季至同期夏季的 海温场和环流场(图3). 可以看到, WNPAC指数为正, 即WNP存在反气旋性环流时, 前期冬、春季WNP呈现 明显的冷海温异常, 这与Wang等(2000)的结论一致, 但 是, 该冷海温异常到夏季时非常弱, 几乎消失, 似乎不 是反气旋维持到夏季的关键因子. 另一方面, WNPAC 维持时, 前期冬、春季和同期夏季 $\mathrm{IO}$ 均呈现暖海温异 常, 其中春季暖海温最为显著, 夏季时暖海温异常有 所减弱.

为进一步分析 $\mathrm{IO}$ 关键区海温与夏季WNPAC的关 系, 我们给出了 $\mathrm{IO}$ 海温指数与夏季WNPAC指数的 17 年滑动相关(图4). 从图中可以看出, 前期冬、春季和 同期夏季 $\mathrm{IO}$ 海温指数与WNPAC指数的相关关系均在 0.5 左右, 前冬IO海温指数与WNPAC关系较为稳定. 而 前期春季和同期夏季与WNPAC关系有所减弱, 且出现 了明显的年代际变化，在2000年之前两者关系稳定， 2000年后相关性有所减弱, 尤其对于夏季IO海温而言, 其与WNPAC的关系在2005年以后关系变得不显著, 说 明IO海温与夏季WNPAC关系在年代际尺度上存在着 


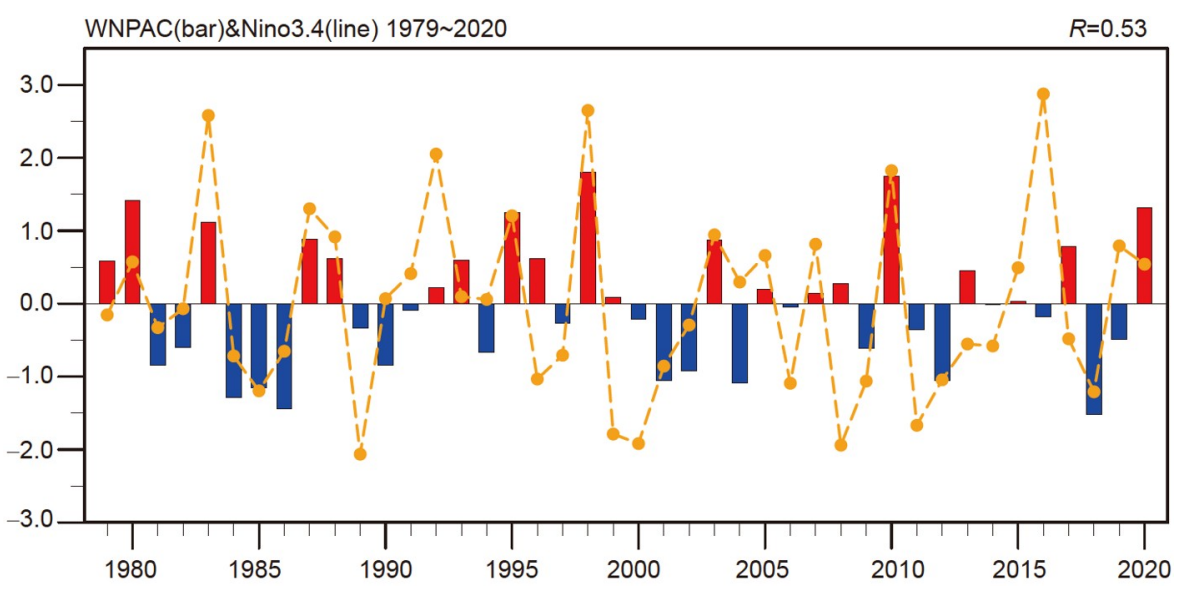

图 1 1979 2020年夏季(JJA)WNPAC指数(柱状)和前冬(DJF)Niño-3.4指数(虚线)的时间序列

JJA: 6月、7月、8月; DJF: 12月、1月、2月

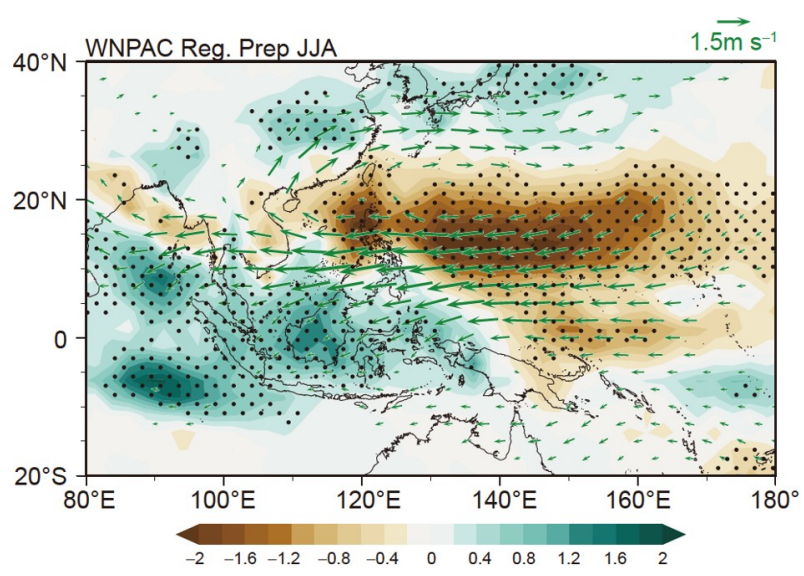

图 2 夏季WNPAC指数回归的同期 $850 \mathrm{hPa}$ 异常降水场(阴 影, 单位: $\mathrm{mm} \mathrm{day}{ }^{-1}$ ) 和风场(矢量, 单位: $\mathrm{m} \mathrm{s}^{-1}$ ) 的空间分布 打点区域表示降水异常通过 $90 \%$ 信度检验，图中仅给出通过 $90 \%$ 显 著性检验的风场

较强的不稳定性. 这不得不使得我们思考一个问题, 即 2000年之后，夏季IO暖海温是否对同期WNPAC的维 持起着重要作用? 我们对比了 2000 年前后夏季 WNPAC指数回归的前期冬春季和同期夏季异常海温 和风场的空间分布(图5), 发现2000年前, WNPAC与前 期冬、春季和同期夏季 $\mathrm{IO}$ 暖海温的正相关关系都比 较显著, 即WNPAC正异常时, IO常出现显著的暖海温 异常, 反之则为冷海温异常. 2000 后它们之间的关系明 显下降，虽然前期冬、春季 $\mathrm{IO}$ 海温还存在一些显著性 信号, 但是显著的范围与 2000 年前相比大大减小; 尤 其是到了夏季, IO海温信号几乎消失, 进一步说明 IO 海温与夏季WNPAC的关系存在年代际尺度的不稳
定性.

接下来，我们将分析夏季 $\mathrm{IO}$ 暖海温与WNPAC指 数在 2000 年前后关系出现不稳定的原因. 从图 5 可以 看出, 夏季WNPAC除了与IO海温存在关系之外, 还与 热带太平洋海温密切相关, 通过对比不难发现, 2000年 前, 夏季WNPAC与同期中东太平洋异常海温的关系 弱, 然而2000年后, 中东太平洋区域出现明显的冷海 温异常. 考虑到两阶段前期冬季热带太平洋地区均存 在显著的暖海温异常, 我们猜想可能是因为 2000 年前 后ENSO事件衰减的快慢对 IO暖海温与夏季WNPAC 关系的稳定性产生了影响.

为了验证我们的猜想, 我们从ENSO事件出发, 给 出了2000年前后两个阶段El Niño衰减年夏季异常海 温场的空间分布(图6), 可以看出, 2000年前, 热带印度 洋同期夏季存在显著的暖海温异常, 而这一信号在 2000年后几乎消失; 与此同时, 2000 年前, 伴随着热带 印度洋的暖海温, 赤道中东太平洋并没有明显的海温 异常; 而2000年后, 热带中东太平洋已转变为负海温 异常, 表现为类La Niña的海温异常特征. 这一结果与 图5 是一致的, 进一步证实夏季 $\mathrm{IO}$ 暖海温与WNPAC关 系的减弱与ENSO事件的衰减速度加快有关. 2000年 前, El Niño事件衰减慢, 到衰减年夏季还未转变为 La Niña状态, IO暖海温响应能持续到夏季, 进而对 WNPAC的维持产生作用; 2000年后, El Niño事件的衰 减速率加快, 到夏季已转为La Niña状态, 不利于IO暖 海温的维持(Feng等, 2014, 2019), 进而削弱了 IO暖海 温与WNPAC的关系, 这可能与IPO在不同位相下对 


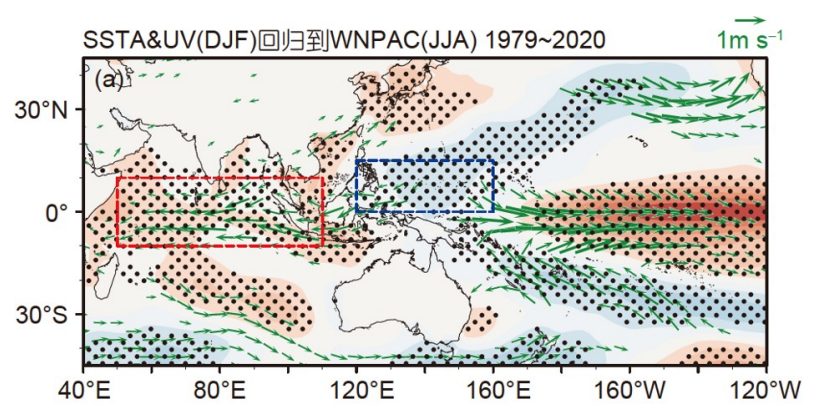

SSTA\&UV(MAM)回归到WNPAC(JJA) 1979 2020 $\quad 1 \overrightarrow{m ~ s}^{-1}$
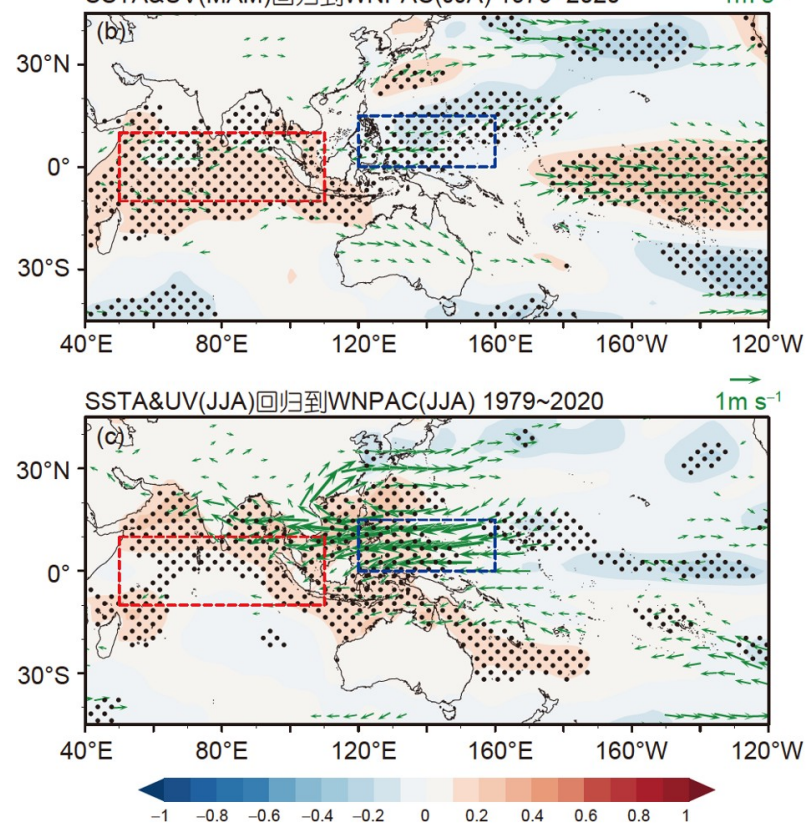

图 $31979 \sim 2020$ 年夏季WNPAC指数回归的前期冬季(a)、 前期春季(MAM)(b)和同期夏季(c)异常海温场(阴影, 单位:

${ }^{\circ} \mathrm{C}$ )和 $850 \mathrm{hPa}$ 风场(矢量, 单位: $\mathrm{m} \mathrm{s}^{-1}$ ) 的空间分布

蓝框表示WNP冷海温的关键区, 红框表示IO暖海温关键区, 打点表 示海温场通过了 $90 \%$ 信度检验的区域，图中仅给出通过 $90 \%$ 信度检 验的风场. MAM: 3月、4月、5月

ENSO衰减速度的调控有关(Liu等, 2021). 值得注意的 是, 2000年后El Nino衰减年夏季赤道中东太平洋表现 出显著的冷海温异常, 这一冷海温异常对夏季WNPAC 的形成和维持也有贡献(Wang等, 2003).

\section{C-mode与WNPAC 的关系}

根据前文的分析可知, IO和WNP海温均似乎不能 很好地解释ENSO衰减年夏季WNP异常环流的维持, 尤其是在2000年后,他们对夏季WNPAC的影响显著减 弱. 前文提到, 近年来研究认为C-mode是WNPAC形成
和维持的重要机制之一，其在ENSO衰减年春-夏季东 亚气候异常中起到了关键作用(Zhang W J等，2015, 2016a，2016b；Jiang等，2019). 下面我们进一步对Cmode与夏季反气旋的关系展开研究. 图7是C-mode指 数与WNPAC指数的 17 年滑动相关. 如图所示, 无论是 前期冬春季, 还是同期夏季, C-mode与夏季WNPAC之 间的正相关关系都很显著，且非常稳定，同期夏季Cmode指数与WNPAC指数的相关关系达到将近 0.7 , 尤 其在 1990 年代中期之后其相关系数达到 0.85 . 即Cmode正位相时, WNP上一般都出现明显的反气旋性环 流, WNPAC指数为正, 而在C-mode负位相时, WNP一 般出现明显的气旋性环流, WNPAC指数为负.

图 8 进一步基于合成分析对比了 2000 年前后 ENSO及C-mode时间演变特征. 由图可知, Niño-3.4指 数始终表现出典型ENSO事件的时间演变特征, 即在 春、夏季发生，在秋、冬季达到成熟，并在衰减年春 季减弱. 与Niño-3.4指数不同的是, C-mode指数 (即热 带太平洋低层风场的第二主分量PC2)在El Niño发展 年的 10 月份仍然为负值, 之后在 12 月迅速转为正值, 在晚冬和早春(FMA)达到峰值, 并且在El Niño衰减年 夏季减弱, 其峰值相对Niño-3.4指数(大致对应PC1)大 约延迟 3 个月左右. 显然, 2000年前后C-mode动力学机 制及其演变特征并没有发生显著变化. 相比于WNP冷 海温和IO暖海温的作用, C-mode对夏季WNPAC维持 的作用始终是较为稳定. 如图9所示, C-mode自身的持 续性较强, 早春与夏季 $\mathrm{C}$-mode指数相关为 0.55 ; 在 ENSO发生的年份, 其相关系数达到 0.65 . 因此基于Cmode, 可以较好地预测北半球夏季的WNPAC及相关 天气气候异常.

\section{6 结论}

本文基于1979 2020年NCEP/NCAR逐月大气环 流和NOAA逐月海表温度资料, 采用相关分析和合成 分析等方法，考察了El Niño衰减年夏季WNPAC的时 间变化特征，并探讨了其维持的可能机制，对比分析 了WNP冷海温、IO暖海温和C-mode在其中的关键作 用, 主要结论如下.

(1) El Niño衰减年夏季WNPAC与前期冬、春季 WNP冷海温存在一定的负相关关系, 但是El Niño衰减 年WNP海温异常信号不能持续到夏季，因而不能很好 


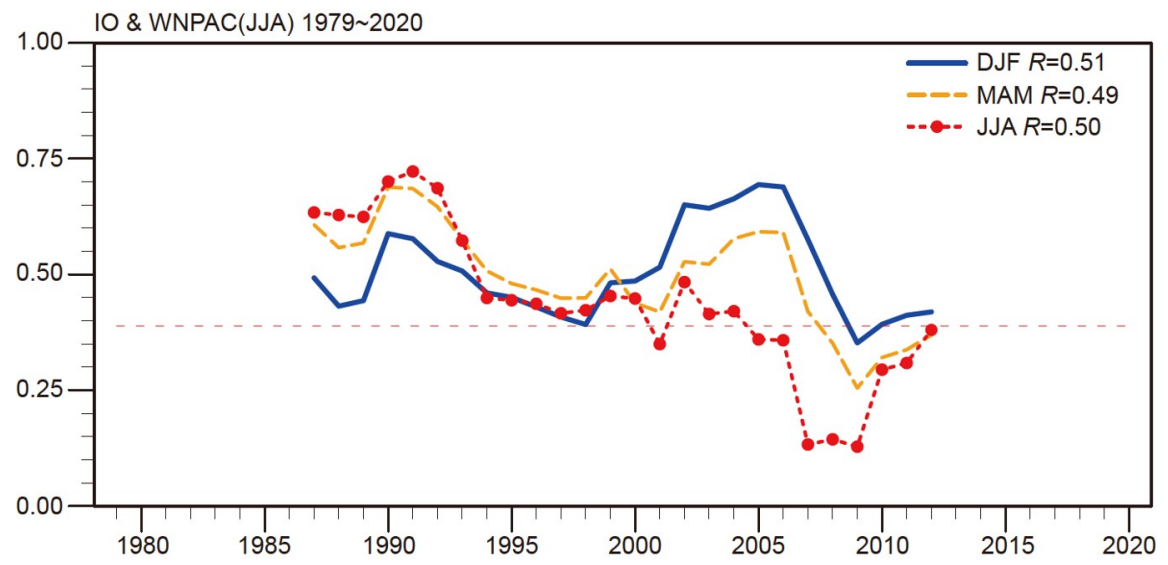

图 4 1979 2020年前期冬季(蓝)、前期春季(橙)和同期夏季(红)IO暖海温关键区指数与夏季WNPAC指数 17年的滑动相关 红色虚线表示 $90 \%$ 信度检验线
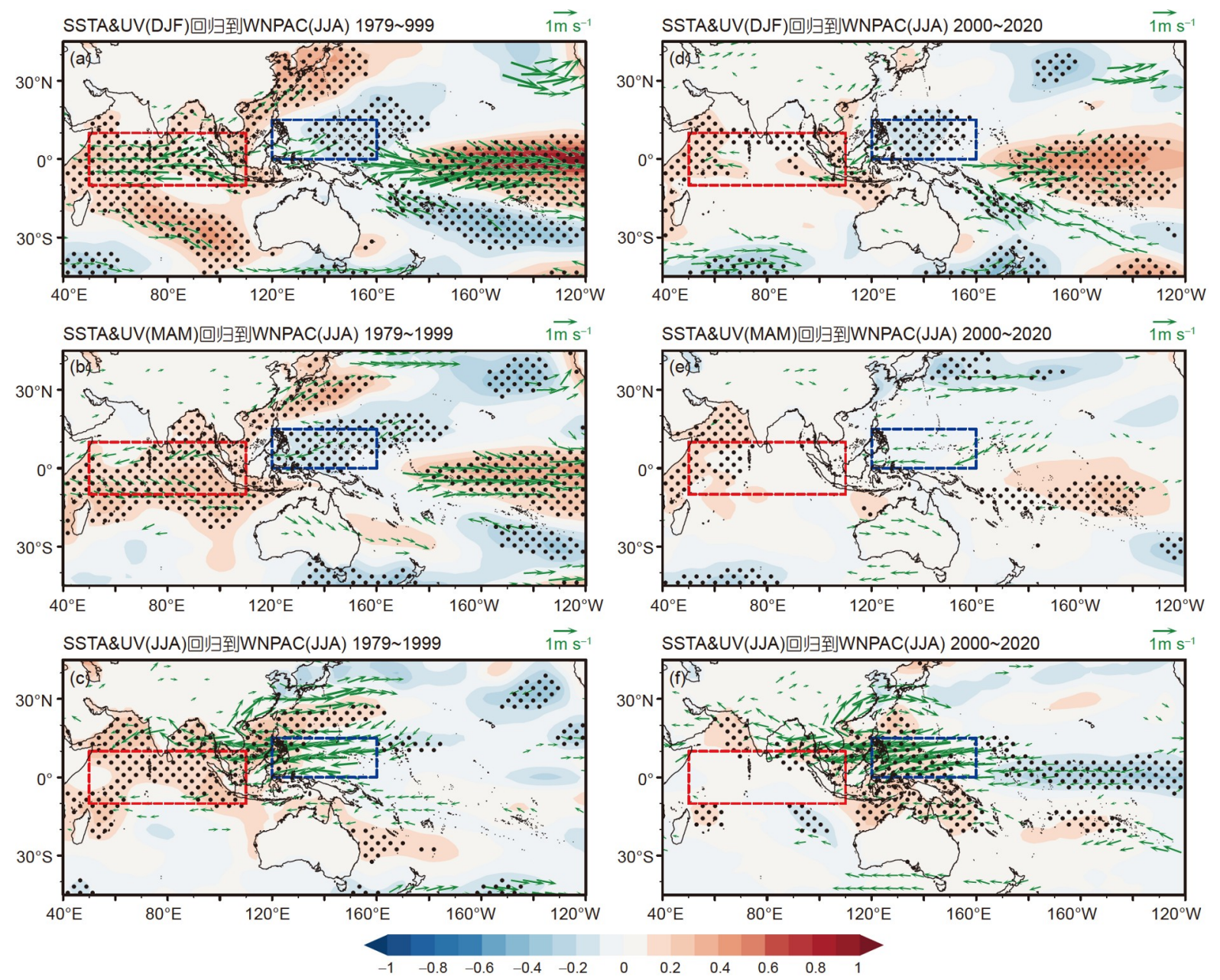

图 5 夏季WNPAC指数回归的前期冬季 $((a),(d))$ 、前期春季 $((b),(e)) 、$ 同期夏季 $((c),(f))$ 异常海温场(矢量, 单位: $\left.{ }^{\circ} C\right)$ 和风场 (阴影, 单位: $\mathrm{m} \mathrm{s}^{-1}$ ) 的空间分布

蓝框表示WNP冷海温关键区, 红框表示IO暖海温关键区, 打点表示海温场通过了 $90 \%$ 信度检验的区域, 图中仅给出通过 $90 \%$ 信度检验的风场. (a) (c), 1979 1999年; (d) (f), 2000 2020年 

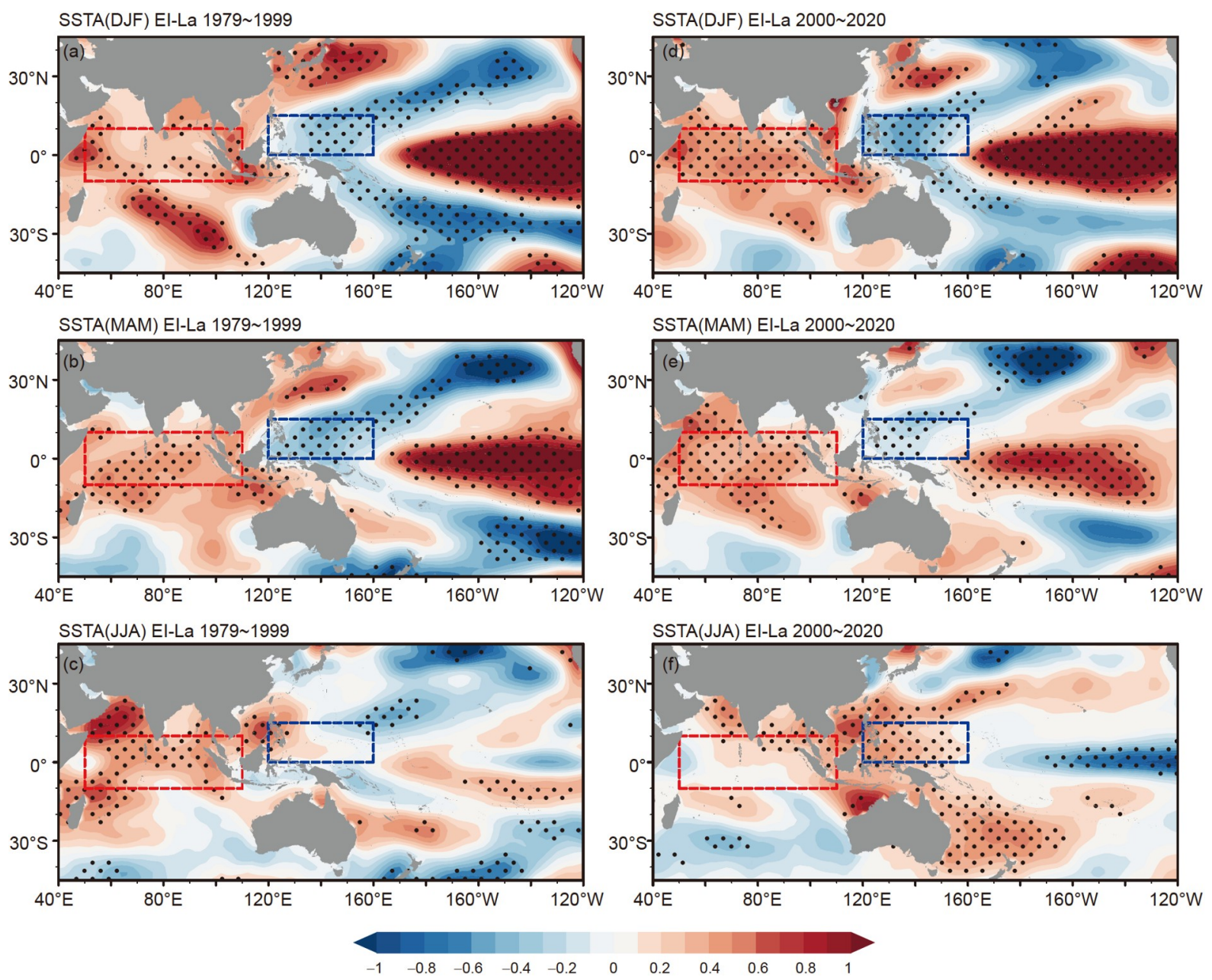

图 6 ENSO衰减年(EI Niño-La Niña)前期冬季 $((a),(d)) 、$ 前期春季 $((b),(e))$ 和同期夏季 $((c),(f))$ 异常海温的合成差值场(阴影, 单位: $\left.{ }^{\circ} \mathrm{C}\right)$

蓝框表示WNP冷海温关键区，红框表示IO暖海温关键区，打点区域表示对应的海温场通过了 90\%信度检验. (a) ( (c)，1979 1999年; (d) (f), 2000 2020年

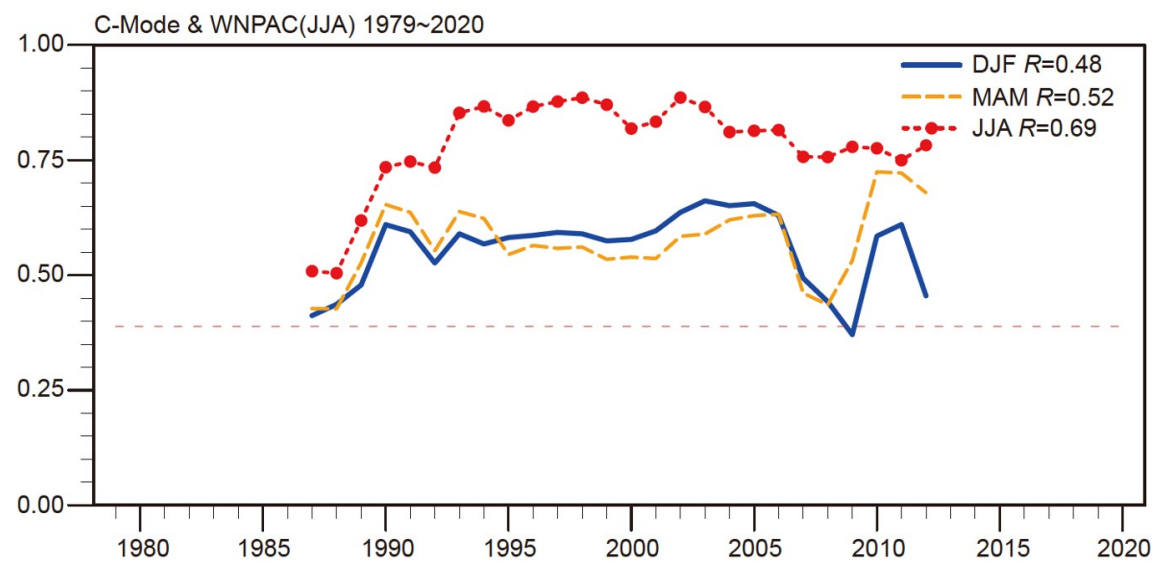

图 7 前期冬季(蓝)、前期春季(橙)和同期夏季(红)C-mode指数与夏季WNPAC指数17年的滑动相关 红色虚线表示 $90 \%$ 信度检验线 

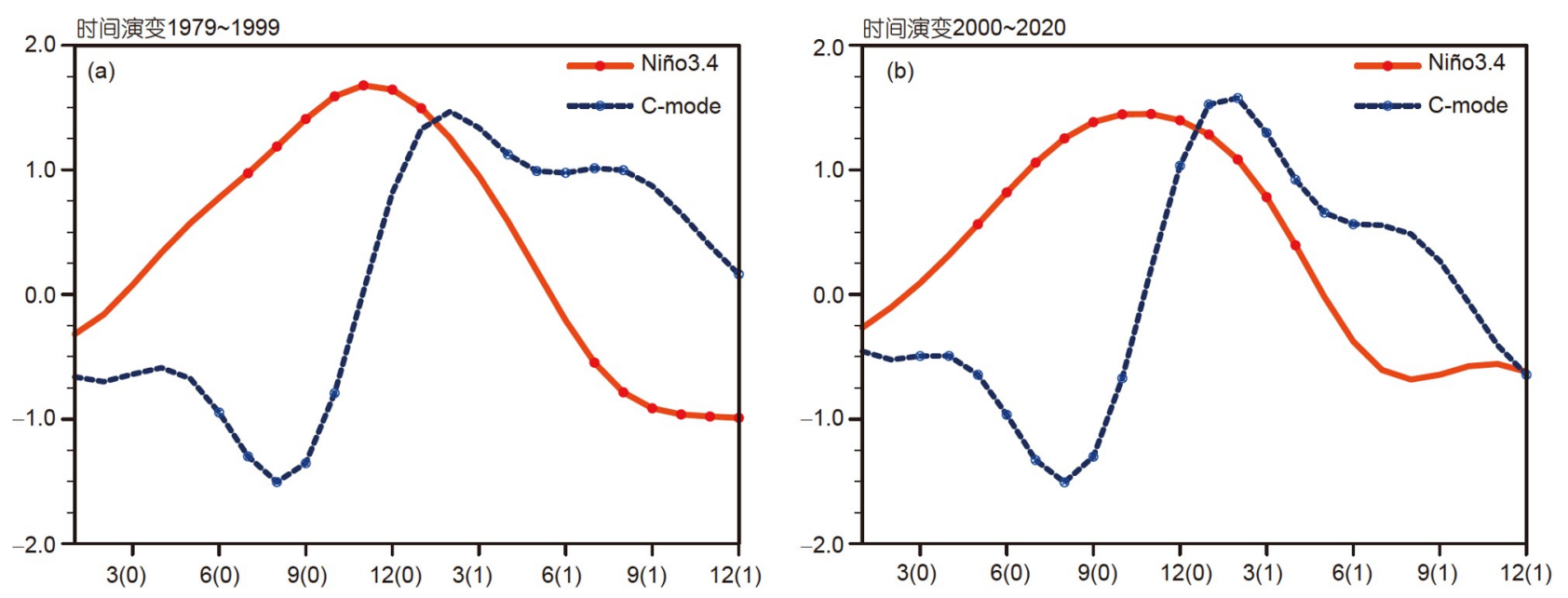

图 8 1979 1999年(a)、2000 2020年(b)EI Niño事件合成的Niño-3.4指数和C-mode指数的时间演变 打点表示通过了 $90 \%$ 的信度检验

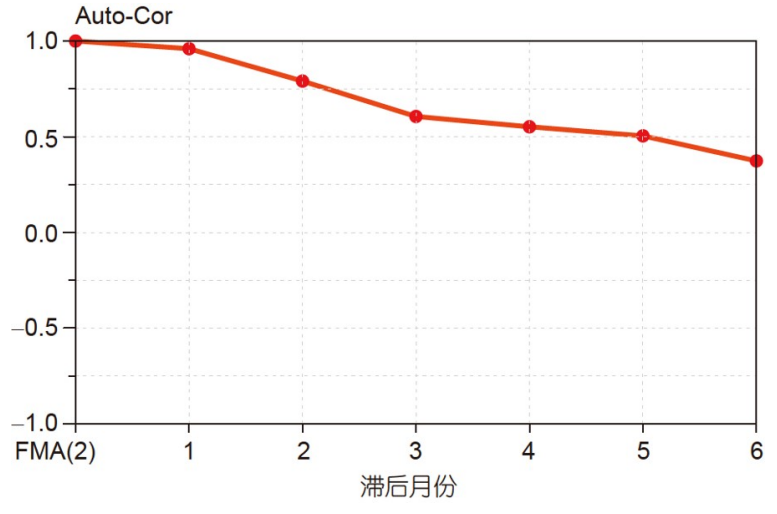

图 9 初春(February-March-April; FMA)C-mode指数的 滞后自相关系数

地解释夏季WNPAC的维持.

(2) IO暖海温与夏季WNPAC之间的正相关关系存 在年代际变化. 在 2000 年前, IO暖海温对WNPAC的关 系能够持续到夏季，2000年后 $\mathrm{IO}$ 暖海温与夏季 WNPAC的关系在统计上不显著. 进一步分析发现，IO 暖海温与WNPAC关系的年代际减弱与El Niño事件衰 减速度的加快有关，2000年前，ENSO事件衰减较慢， 到夏季时还未转变为La Niña状态, IO海温存在显著的 暖异常响应，因而能对夏季WNPAC的维持产生作用; 2000年后，El Niño事件衰减速率加快，热带中东太平 洋在夏季时已转为La Niña状态, IO暖海温响应也消失, 因此对夏季WNPAC的维持作用也明显减弱.
(3) 与WNP冷海温和IO暖海温相比, C-mode与夏 季WNPAC的正相关关系在年代际尺度上较稳定, 在El Niño衰减年夏季WNP异常环流的维持中起到了关键 的作用. 在当前气候变化的背景下，基于C-mode预报 北半球夏季WNPAC变率具有优越性.

\section{参考文献}

黄刚, 胡开明. 2008. 夏季北印度洋海温异常对西北太平洋低层反气 旋异常的影响. 大气科学学报, 31: 749-757

黄平, 黄荣辉. 2010. E1 Niño事件对其衰减阶段夏季中国降水季节内 演变的影响及其机理. 大气科学学报, 33: 513-519

李海燕, 张文君, 何金海. 2016. ENSO及其组合模态对中国东部各季 节降水的影响. 气象学报, 74: 322-334

陶诗言, 张庆云. 1998. 亚洲冬夏季风对ENSO事件的响应. 大气科学, 22: $399-407$

张人禾, 闵庆烨, 苏京志. 2017. 厄尔尼诺对东亚大气环流和中国降 水年际变异的影响: 西北太平洋异常反气旋的作用. 中国科学: 地球科学, 47: 544-553

周天军, 俞永强, 宇如聪, 张学洪, 李肇新. 2004. 印度洋对ENSO事件 的响应: 观测与模拟. 大气科学, 28: 357-373

Alexander M A, Bladé I, Newman M, Lanzante J R, Lau N C, Scott J D. 2002. The atmospheric bridge: The influence of ENSO teleconnections on air-sea interaction over the global oceans. J Clim, 15: 22052231

Bjerknes J. 1969. Atmospheric teleconnections from the equatorial Pacific. Mon Weather Rev, 97: 163-172 
Chang C P, Zhang Y S, Li T. 2000a. Interannual and interdecadal variations of the East Asian summer monsoon and tropical Pacific SSTs. Part I: Roles of the subtropical ridge. J Clim, 13: 4310-4325

Chang C P, Zhang Y, Li T. 2000b. Interannual and interdecadal variations of the East Asian summer monsoon and tropical Pacific SSTs. Part II: Meridional structure of the monsoon. J Clim, 13: $4326-4340$

Chen W, Graf H F, Huang R H. 2000. The interannual variability of East Asian winter monsoon and its relation to the summer monsoon. Adv Atmos Sci, 17: 48-60

Du Y, Xie S P, Huang G, Hu K M. 2009. Role of air-sea interaction in the long persistence of El Niño-induced north Indian Ocean warming. J Clim, 22: 2023-2038

Feng J, Li J P, Jin F F, Liu Z Y, Zhao S. 2019. Effect of El Niño on the response ratio of Hadley circulation to different SST meridional structures. Clim Dyn, 53: 3877-3891

Feng J, Wang L, Chen W. 2014. How does the East Asian summer monsoon behave in the decaying phase of El Niño during different PDO phases? J Clim, 27: 2682-2698

Gill A E. 1980. Some simple solutions for heat-induced tropical circulation. Q J R Met Soc, 106: 447-462

Harrison D E, Larkin N K. 1996. The COADS sea level pressure signal: A near-global El Niño composite and time series view, 1946-1993. J Clim, 9: 3025-3055

Huang B, Thorne P W, Banzon V F, Boyer T, Chepurin G, Lawrimore J H, Menne M J, Smith T M, Vose R S, Zhang H M. 2017. Extended reconstructed sea surface temperature, version 5 (ERSSTv5): Upgrades, validations, and intercomparisons. J Clim, 30: 8179-8205

Jiang F, Zhang W J, Geng X, Stuecker M F, Liu C. 2019. Impacts of central Pacific El Niño on southern China spring precipitation controlled by its longitudinal position. J Clim, 32: 7823-7836

Kanamitsu M, Ebisuzaki W, Woollen J, Yang S K, Hnilo J J, Fiorino M, Potter G L. 2002. Ncep-doe amip-ii reanalysis (r-2). Bull Amer Meteorol Soc, 83: 1631-1644

Klein S A, Soden B J, Lau N C. 1999. Remote sea surface temperature variations during ENSO: Evidence for a tropical atmospheric bridge. J Clim, 12: 917-932

Li T, Wang B, Wu B, Zhou T, Chang C P, Zhang R. 2017. Theories on formation of an anomalous anticyclone in western north Pacific during El Niño: A review. J Meteorol Res, 31: 987-1006

Lin Z D, Lu R Y. 2009. The ENSO's effect on eastern China rainfall in the following early summer. Adv Atmos Sci, 26: 333-342

Liu F Y, Zhang W J, Jin F F, Hu S Q. 2021. Decadal modulation of the ENSO-Indian Ocean basin warming relationship during the decaying summer by the interdecadal Pacific oscillation. J Clim, 34: 2685-2699
Matsuno T. 1966. Quasi-geostrophic motions in the equatorial area. J Meteorol Soc Jpn, 44: 25-43

McGregor S, Ramesh N, Spence P, England M H, McPhaden M J, Santoso A. 2013. Meridional movement of wind anomalies during ENSO events and their role in event termination. Geophys Res Lett, 40: 749-754

McGregor S, Timmermann A, Schneider N, Stuecker M F, England M H. 2012. The effect of the south Pacific convergence zone on the termination of El Niño events and the meridional asymmetry of ENSO. J Clim, 25: 5566-5586

Stuecker M F, Jin F F, Timmermann A, McGregor S. 2015. Combination mode dynamics of the anomalous northwest Pacific anticyclone. J Clim, 28: 1093-1111

Stuecker M F, Timmermann A, Jin F F, McGregor S, Ren H L. 2013. A combination mode of the annual cycle and the El Niño/Southern Oscillation. Nat Geosci, 6: 540-544

Wallace J M, Gutzler D S. 1981. Teleconnections in the geopotential height field during the Northern Hemisphere winter. Mon Weather Rev, 109: 784-812

Wallace J M, Rasmusson E M, Mitchell T P, Kousky V E, Sarachik E S, von Storch H. 1998. On the structure and evolution of ENSO-related climate variability in the tropical Pacific: Lessons from TOGA. J Geophys Res, 103: 14241-14259

Wang B, Li J, He Q. 2017. Variable and robust East Asian monsoon rainfall response to El Niño over the past 60 years (1957-2016). Adv Atmos Sci, 34: 1235-1248

Wang B, Wu R G, Fu X H. 2000. Pacific-East Asian teleconnection: How does ENSO affect East Asian climate? J Clim, 13: 1517-1536

Wang B, Wu R G, Li T. 2003. Atmosphere-warm ocean interaction and its impacts on Asian-Australian monsoon variation. J Clim, 16: $1195-1211$

Wang B, Xiang B Q, Lee J Y. 2013. Subtropical high predictability establishes a promising way for monsoon and tropical storm predictions. Proc Natl Acad Sci USA, 110: 2718-2722

Wang C Y, Xie S P, Kosaka Y. 2018. Indo-western Pacific climate variability: ENSO forcing and internal dynamics in a tropical Pacific pacemaker simulation. J Clim, 31: 10123-10139

Wang C Y, Xie S P, Kosaka Y. 2020. ENSO-unrelated variability in Indo-Northwest Pacific climate: Regional coupled Ocean-Atmospheric feedback. J Clim, 33: 4095-4108

Wu B, Li T, Zhou T. 2010. Relative contributions of the Indian Ocean and local SST anomalies to the maintenance of the western North Pacific anomalous anticyclone during the El Niño decaying summer.. J Clim, 23: 2974-2986

Xie P P, Arkin P A. 1997. Global precipitation: A 17-year monthly analysis based on gauge observations, satellite estimates, and 
numerical model outputs. Bull Amer Meteorol Soc, 78: 2539-2558

Xie S P, Hu K M, Hafner J, Tokinaga H, Du Y, Huang G, Sampe T. 2009. Indian Ocean capacitor effect on Indo-western Pacific climate during the summer following El Niño. J Clim, 22: 730-747

Xie S P, Kosaka Y, Du Y, Hu K M, Chowdary J S, Huang G. 2016. Indo-western Pacific Ocean capacitor and coherent climate anomalies in post-ENSO summer: A review. Adv Atmos Sci, 33: $411-432$

Yang J L, Liu Q Y, Xie S P, Liu Z Y, Wu L X. 2007. Impact of the Indian Ocean SST basin mode on the Asian summer monsoon. Geophys Res Lett, 34: L02708

Zhang R H, Li T R, Wen M, Liu L K. 2015. Role of intraseasonal oscillation in asymmetric impacts of El Niño and La Niña on the rainfall over southern China in boreal winter. Clim Dyn, 45: 559567

Zhang R H, Sumi A, Kimoto M. 1996. Impact of El Niño on the East Asian Monsoon. J Meteorol Soc Jpn, 74: 49-62
Zhang R H, Sumi A, Kimoto M. 1999. A diagnostic study of the impact of El Niño on the precipitation in China. Adv Atmos Sci, 16: 229241

Zhang R H, Sumi A. 2002. Moisture circulation over East Asia during El Niño episode in northern Winter, Spring and Autumn. J Meteorol Soc Jpn, 80: 213-227

Zhang W J, Jin F F, Stuecker M F, Wittenberg A T, Timmermann A, Ren H L, Kug J S, Cai W J, Cane M. 2016a. Unraveling El Niño's impact on the East Asian monsoon and Yangtze River summer flooding. Geophys Res Lett, 43: 11,375-11,382

Zhang W J, Li H Y, Jin F F, Stuecker M F, Turner A G, Klingaman N P. 2015. The annual-cycle modulation of meridional asymmetry in ENSO's atmospheric response and its dependence on ENSO zonal structure. J Clim, 28: 5795-5812

Zhang W J, Li H Y, Stuecker M F, Jin F F, Turner A G. 2016b. A new understanding of El Niño's impact over East Asia: Dominance of the ENSO combination mode. J Clim, 29: 4347-4359

(责任编委: 陈文) 